\title{
Silent cerebral infarction is associated with the development and progression of nephropathy in patients with type 2 diabetes
}

\author{
Ryotaro Bouchi, Tetsuya Babazono, Naoshi Yoshida, Izumi Nyumura, Kiwako Toya, Toshihide Hayashi, \\ Ko Hanai, Nobue Tanaka, Akiko Ishii and Yasuhiko Iwamoto
}

Chronic kidney disease (CKD) is an important risk factor for cardiovascular disease in patients with diabetes. The relationship between renal manifestations of CKD (albuminuria and decreased glomerular filtration rate) and silent cerebral infarction (SCI) has attracted attention; however, most studies examined the effects of components of CKD on prevalence of SCI. We sought to assess the relationship between $\mathrm{SCl}$ and the development and progression of nephropathy in type 2 diabetic patients. We studied 366 type 2 diabetic patients with normoalbuminuria (urinary albumin-to-creatinine ratio [ACR] $<30 \mathrm{mg} \mathrm{g}^{-1}, N=246$ ) or microalbuminuria (ACR $=30-299 \mathrm{mg} \mathrm{g}^{-1}, N=120$ ). SCl was defined by cranial MRI. The primary end point was progression from normo- to microalbuminuria or from micro- to macroalbuminuria. The cumulative incidence of the primary end point was estimated using the Kaplan-Meier method. Risk estimates for reaching the end point were calculated using Cox proportional hazard model analyses. During a median follow-up period of 3.9 years, 23 normoalbuminuric and 24 microalbuminuric patients reached the primary end point. Patients with $\mathrm{SCl}(N=171)$ had a greater incidence of reaching the end point than those without $\mathrm{SCl}(N=195, P=0.020$ by the log-rank test), with a hazard ratio of $2.02(95 \%$ confidence interval $=1.09-3.72, P=0.025)$ in the multivariate Cox regression model. Although the common pathogenesis of $\mathrm{SCl}$ and albuminuria in diabetic patients is still unclear, $\mathrm{SCl}$ may be a predictor of progression of nephropathy in type 2 diabetic patients.

Hypertension Research (2010) 33, 1000-1003; doi:10.1038/hr.2010.122; published online 8 July 2010

Keywords: albuminuria; diabetic nephropathy; silent cerebral infarction

\section{INTRODUCTION}

Chronic kidney disease $(\mathrm{CKD})^{1}$ is an independent risk factor for cardiovascular disease in both the general population ${ }^{2}$ and diabetic patients. $^{3}$ Albuminuria, one of the important components of CKD, ${ }^{1}$ has been shown to be associated with incident cardiovascular disease ${ }^{3,4}$ and progression of renal impairment. ${ }^{5}$ It is, therefore, widely recommended that urinary albumin should be measured not only for the early detection of diabetic nephropathy, ${ }^{6,7}$ but also to evaluate the risk of incident cardiovascular disease.

Silent cerebral infarction (SCI), which has a predilection for the subcortical white matter and basal ganglia, is commonly observed on cranial MRI scans in the elderly and hypertensive patients. ${ }^{8,9} \mathrm{SCI}$ is thought to be a clinical end-organ manifestation of arteriosclerosis in the brain, as well as retinal arterial sclerosis in the eyes and renal sclerosis in the kidneys, ${ }^{10,11}$ and the presence of SCI predicts incident clinically evident stroke, ${ }^{12}$ cardiovascular disease ${ }^{13}$ and dementia. ${ }^{14}$ SCI has been also shown to be associated with albuminuria in community-dwelling elderly persons ${ }^{15}$ and diabetic patients. ${ }^{16}$ We have recently shown that (micro)albuminuria may be a predictor of incident stroke in patients with type 2 diabetes. ${ }^{17}$ These studies suggest the organ cross-talk between brain and kidneys. A recent study indicated that SCI may predict progression to end-stage renal failure in patients with type 2 diabetes; ${ }^{18}$ however, there is limited understanding of whether patients with SCI are at increased risk of the development and progression of early stage of diabetic nephropathy. We, therefore, sought to determine the potential contribution of SCI to increased risk of development and progression of nephropathy in patients with type 2 diabetes.

\section{METHODS}

Study population

This study was conducted in accordance with the Declaration of Helsinki. We recruited consecutive patients with type 2 diabetes, 20 years or older, who underwent cranial MRI at the Diabetes Center, Tokyo Women's Medical University Hospital, Tokyo, Japan, during the period between 1 July 2003 and 30 April 2008. As the Japan Brain Dock Society recommends brain MRI scans in high-risk middle-aged or elderly persons (that is those with hypertension or diabetes) (http://www.snh.or.jp/jsbd/pdf/guideline2008.pdf), 
we generally performed MRI for the purpose of screening for SCI in this study. Patients with type 1 diabetes, patients with macroalbuminuria (as defined later) and undergoing renal replacement therapy, pregnant women and patients with infectious or malignant diseases were excluded. Those who had clinical and laboratory missing data were also excluded. Type 2 diabetes was diagnosed according to the criteria of the World Health Organization definition. ${ }^{19}$

Participants underwent a routine medical history, physical examination and blood sampling. Information regarding smoking and family history of cardiovascular disease was obtained using a standard questionnaire. Smoking history was classified as either current smoker or non-smoker. Physical examination included blood pressure measurement and anthropometry; laboratory examinations included hemoglobin $\mathrm{AlC}$, serum lipids and creatinine and urinary albumin excretion.

History of cerebrovascular disease was defined as earlier history of transient ischemic attack and/or stroke. History of coronary artery disease was defined as the presence of any of the following conditions: angina pectoris diagnosed by coronary angiography or myocardial scintigraphy, earlier myocardial infarction or earlier revascularization. History of peripheral artery disease was defined as earlier lower extremity peripheral artery disease according to American College of Cardiology/American Heart Association 2005 guideline. ${ }^{20}$

\section{Measurements}

Cranial MRI scans were performed using a 1.5-T MR system (GyroScan Intere 1.5T master, Philips or MRT-2001/P3 excelart, Toshiba). T1- (repetition time, $400 \mathrm{~ms}$; echo time, $10 \mathrm{~ms}$ ) and T2-weighted images (repetition time, $3500 \mathrm{~ms}$; echo time, $90 \mathrm{~ms}$ ) were obtained in the transverse plane with $7-\mathrm{mm}$ thick sections. SCI was defined by cranial MRI examinations as an area of low-signal intensity measuring at least $3 \mathrm{~mm}$ on T1-weighted images, which was also visible as a hyperintense lesion on T2-weighted images, according to the clinical guidelines by the Japanese Society for Detection of Asymptomatic Brain Disease. ${ }^{21}$ Hyperintense punctuate lesions evident only on T2-weighted images were excluded from the diagnosis of SCI. In each case, SCI was diagnosed by consensus of two neuroradiologists at the Tokyo Women's Medical University Hospital.

Classification of the degree of urinary albumin was assessed according to American Diabetes Association criteria, on the basis of at least two out of three albumin-to-creatinine ratio (ACR) measurements obtained from first morning urine specimens. ${ }^{7}$ Normo-, micro- and macroalbuminuria were defined as an ACR $<30,30-299$ and $\geqslant 300 \mathrm{mg} \mathrm{g}^{-1}$, respectively. Glomerular filtration rate (GFR) was estimated using the following modified three-variable equation for Japanese: $\mathrm{GFR}=194 \times \mathrm{SCr}^{-1.094} \times \mathrm{age}^{-0.287}([$ if female $] \times 0.739) \mathrm{ml} \mathrm{min}^{-1}$ $1.73 \mathrm{~m}^{-2} .22$ The primary end point was defined as transition from normoto microalbuminuria or micro- to macroalbuminuria, confirmed on at least two consecutive urinary ACR measurements to reduce misclassification.

Hemoglobin A1C was measured by high-performance liquid chromatography (normal range: $4.3-5.8 \%$ ), and total cholesterol and high-density lipoprotein cholesterol were determined enzymatically. Low-density lipoprotein cholesterol was calculated using the Friedewald equation when serum triglycerides level was $<400 \mathrm{mg}$ per $100 \mathrm{ml}^{23}$

\section{Statistical analyses}

Data were expressed as percentage, arithmetic mean \pm s.d. or geometric mean with $95 \%$ confidence interval (CI), as appropriate according to data distribution. Triglycerides and ACR were logarithmically transformed because of skewed distributions. For statistical analyses, Student's $t$-test or $\chi^{2}$ test were conducted according to the appropriate situation. The cumulative incidence of the primary end point was estimated using the Kaplan-Meier method. Risk estimates for reaching the end point were calculated using univariate and multivariate Cox proportional hazard model analyses. The following variables were incorporated as covariates: age, gender, duration of diabetes, presence of proliferative diabetic retinopathy, smoking status, body mass index, systolic and diastolic blood pressure, use of renin-angiotensin system inhibitors (angiotensin-converting enzyme inhibitors and angiotensin type 1-receptor blockers), antiplatelet agents, statins, hemoglobin A1C, triglycerides, high-density lipoprotein cholesterol, low-density lipoprotein cholesterol, hemoglobin, uric acid, eGFR and urinary ACR at baseline. Prognostic factors were selected using the stepwise procedure, specifying the significant levels for entering another explanatory variable into the model as 0.25 , and that for removing an explanatory variable from the model as 0.15 . A $P$-value $<0.05$ was considered significant. All statistical analyses were performed using the SAS version 9.13 (SAS Institute, Cary, NC, USA).

\section{RESULTS}

A total of 366 type 2 diabetic patients had sufficient baseline and follow-up data to qualify for inclusion, including 149 women and 217 men, with a mean ( \pm s.d.) age of $64 \pm 10$ years (range: $29-89$ years). At baseline, 246 patients had normoalbuminuria and 120 patients had microalbuminuria. SCI was detected in 171 patients (46.7\%). Patients with SCI were older and had higher systolic blood pressure and urinary albumin excretion and lower body mass index, hemoglobin levels and eGFR than patients without SCI (Table 1).

During a median follow-up period of 3.9 years (range: 0.3-6.0 years), 23 normoalbuminuric and 24 microalbuminuric patients reached the primary end point.

Twelve $(8.5 \%)$ of 141 patients without SCI and $11(10.5 \%)$ of 105 patients with SCI progressed from normo- to microalbuminuria. The progression from micro- to macroalbuminuria was observed in 6 $(11.1 \%)$ of 54 patients without SCI and $18(27.3 \%)$ of 66 patients with SCI. In contrast, the regression of nephropathy (from micro- to

Table 1 Demographic and laboratory data in type 2 diabetic patients with and without $\mathrm{SCl}$ at baseline

\begin{tabular}{lccr}
\hline & $\begin{array}{c}\text { Without SCl } \\
(\mathrm{N}=195)\end{array}$ & $\begin{array}{c}\text { With SCl } \\
(\mathrm{N}=171)\end{array}$ & P-value \\
\hline Age (years) & $61 \pm 10$ & $69 \pm 8$ & $<0.00$ \\
Gender (\% men) & 61.0 & 57.3 & 0.470 \\
Current smoker (\%) & 22.6 & 14.6 & 0.053 \\
Diabetes duration (years) & $15 \pm 10$ & $15 \pm 9$ & 0.76 \\
Body mass index (kg m${ }^{-2}$ ) & $25.1 \pm 4.0$ & $24.1 \pm 3.4$ & 0.01 \\
Systolic blood pressure (mm Hg) & $134 \pm 19$ & $139 \pm 19$ & 0.008 \\
Diastolic blood pressure (mm Hg) & $77 \pm 12$ & $75 \pm 14$ & 0.112 \\
Proliferative diabetic retinopathy (\%) & 16.4 & 18.7 & 0.563
\end{tabular}

\begin{tabular}{|c|c|c|c|}
\hline \multicolumn{4}{|l|}{ Medication (\%) } \\
\hline OHA/insulin & $54.9 / 39.5$ & $55.6 / 40.9$ & 0.778 \\
\hline RAS blockers (\%) & 45.1 & 55.6 & 0.048 \\
\hline Antiplatelet agents (\%) & 34.9 & 54.4 & $<0.001$ \\
\hline Statins (\%) & 33.3 & 34.5 & 0.814 \\
\hline Hemoglobin A1C (\%) & $8.1 \pm 1.6$ & $7.9 \pm 1.6$ & 0.316 \\
\hline Triglycerides $\left(\mathrm{mmol}^{-1}\right)^{\mathrm{b}}$ & $1.41(1.31-1.52)$ & $1.36(1.26-1.48)$ & 0.508 \\
\hline HDL cholesterol (mmol $\left.{ }^{-1}\right)$ & $1.31 \pm 0.41$ & $1.35 \pm 0.43$ & 0.414 \\
\hline LDL cholesterol (mmol $\left.\mathrm{l}^{-1}\right)$ & $3.01 \pm 0.85$ & $2.97 \pm 0.77$ & 0.604 \\
\hline Uric acid $\left(\mu \mathrm{mol} \mathrm{I}^{-1}\right)$ & $304 \pm 73$ & $300 \pm 85$ & 0.649 \\
\hline Hemoglobin $\left(\mathrm{gl}^{-1}\right)$ & $141 \pm 13$ & $135 \pm 15$ & $<0.001$ \\
\hline$A C R\left(m g g^{-1}\right)^{\mathrm{b}}$ & $18(15-21)$ & $24(20-29)$ & 0.007 \\
\hline$<30(\%)$ & 72.3 & 61.4 & 0.027 \\
\hline 30-299 (\%) & 27.7 & 38.6 & 0.027 \\
\hline Serum creatinine $\left(\mu \mathrm{mol} \mathrm{I}{ }^{-1}\right)$ & $65.6 \pm 14.9$ & $72.1 \pm 22.5$ & 0.001 \\
\hline eGFR ( $\left.\mathrm{ml} \mathrm{min}-11.73 \mathrm{~m}^{-2}\right)$ & $77.9 \pm 17.0$ & $68.6 \pm 16.8$ & $<0.001$ \\
\hline
\end{tabular}

Abbreviations: ACR, albumin-to-creatinine ratio; $\mathrm{Cl}$, confidence interval; GFR, glomerular filtration rate; HDL, high-density lipoprotein; LDL, low-density lipoprotein; OHA, oral hypoglycemic agent; RAS, rennin-angiotensin system; $\mathrm{SCl}$, silent cerebral infarction. Data are expressed as mean \pm s.d., geometric mean $(95 \% \mathrm{Cl})$, or percentage.

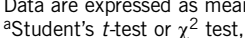
a Student's $t$-test or
bGeometric mean. 


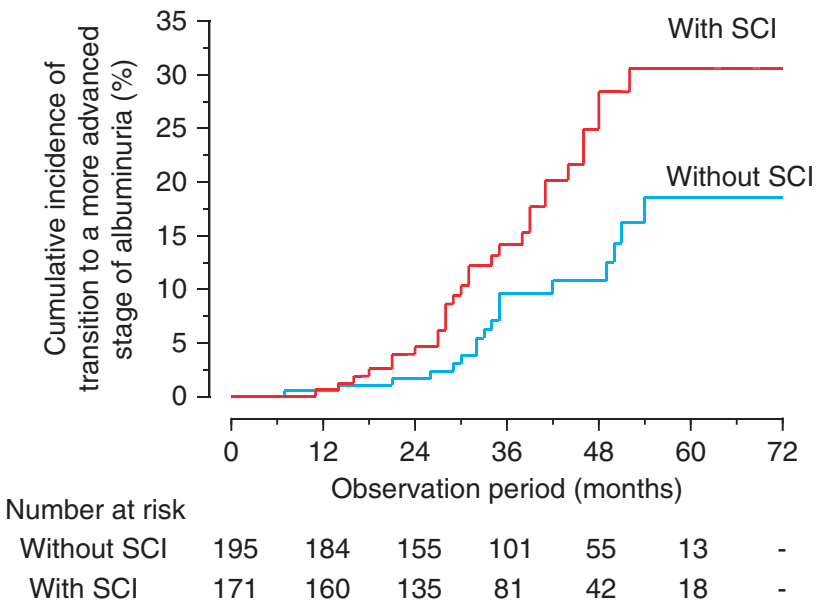

Figure 1 Cumulative incidence of the end point (transition to a more advanced stage of albuminuria) in Japanese type 2 diabetic patients with and without $\mathrm{SCl}$. The difference between Kaplan-Meier estimates for the two groups was statistically significant by log-rank test $(P=0.020)$.

normoalbuminuria) was also observed in 12 (10.0\%) of 120 microalbuminuric patients during the follow-up period. The cumulative incidence of the primary end point, that is progression to micro- or macroalbuminuria, was significantly higher for patients with SCI than those without SCI (log-rank test, $P=0.020$; Figure 1). In the univariate Cox proportional hazard model, the hazard ratio of the end point for patients with SCI $v s$. those without SCI was 1.98 (95\% CI=1.10-3.56, $P=0.023)$. This statistical association remained significant even after adjustment for other covariates using the stepwise multivariate Cox regression analysis (hazard ratio $=2.02,95 \% \mathrm{CI}=1.09-3.72, P=0.025$ ). Other covariates finally included in the multivariate analysis were SCI, logarithmically transformed ACR, hemoglobin and proliferative diabetic retinopathy. SCI (hazard ratio $=2.02,95 \% \mathrm{CI}=1.09-3.72$, $P=0.025$ ), logarithmically transformed ACR (hazard ratio=4.61, 95\% CI $=2.65-8.02, P<0.001$ ) and hemoglobin (hazard ratio=0.843, $95 \% \mathrm{CI}=0.71-0.94, P=0.016)$ were selected as significant covariates.

\section{DISCUSSION}

Albuminuria is a strong risk factor for cardiovascular disease; ${ }^{3,4}$ however, limited data are available on the opposite relation, for example whether patients with cardiovascular disease have a great risk of incident albuminuria. ${ }^{18,24}$ In this study, we observed a higher incidence of the development and progression of nephropathy in type 2 diabetic subjects with SCI than without. This association remained significant after adjustment for covariates. To the best of our knowledge, this is the first study to show the significant association between SCI and the development or progression of albuminuria in a cohort of diabetic patients.

Concerning the cardio-renal association in diabetic patients, as we discussed earlier, ${ }^{17}$ albuminuria and cardiovascular disease share numerous risk factors that may help to explain the observed relationship between SCI and nephropathy in this study. Hemodynamic and anatomical aspects of renal and cerebral small vessels share many similarities, ${ }^{25,26}$ and a common pathogenesis may contribute to both SCI and incident nephropathy in patients with type 2 diabetes. Another possible mechanism that may contribute to the association of SCI with albuminuria involves asymmetric dimethylarginine, an endogenous inhibitor of nitric oxide synthase, which was shown to be associated with prevalent SCI in the Framingham offspring study. ${ }^{27}$
We have recently shown that elevated plasma asymmetric dimethylarginine is a significant predictor of the development and progression of nephropathy in diabetic patients. ${ }^{28}$ Furthermore, Hashimoto et al. ${ }^{29}$ cross-sectionally showed that large artery stiffness (pulse wave velocity) is an important factor, which explains the association between cerebral lacunar infarction and albuminuria. Stiffening of large arteries increases the pulsatile stress because of increased aortic characteristic impedance and early return of wave reflection, and it could eventually lead to microvascular damage of the brain (SCI) and kidneys (albuminuria). Therefore, the cerebro-renal association (SCI and albuminuria) in this study may be at least in part explained by stiffening of large arteries. Further studies are needed to better understand the common pathogenesis of SCI and albuminuria in diabetic patients.

In contrast to our study, Uzu et al. ${ }^{18}$ recently showed that SCI was not associated with the incidence of nephropathy, but progression to renal failure in Japanese diabetic patients. The differences of the impact of SCI on the renal outcomes between the study ${ }^{18}$ and ours are unclear. One possible explanation may be due to the differences in the patient characteristics. In our study, the prevalence of patients with microalbuminuria was nearly equal to that in the population-based study in Japan, ${ }^{30}$ but smaller than that of the study by Uzu et al. ${ }^{18}$ In addition, patients in this study were older, probably explaining the higher prevalence of SCI because aging is a strong predictor of SCI. ${ }^{8}$ Further large prospective studies are needed to elucidate the association between SCI and incident albuminuria in diabetic patients.

Our study population was ethnically and socially homogeneous and, therefore, may not be generalizable to other ethnic groups or the general population. Furthermore, cranial MRIs in this study were mainly performed for the purpose of screening for SCI, yielding a strong selection bias. Therefore, the higher prevalence of SCI in this study may be overestimated. Second, the follow-up period is relatively short. Third, we never determined the impact of SCI on the progression to end-stage renal disease. Nevertheless, this study is the first to reveal the significant association between SCI and incident albuminuria in diabetic patients and may facilitate identification of diabetic patients carrying a higher risk of progression of nephropathy.

In conclusion, SCI may be a predictor of the development and progression of nephropathy in Japanese patients with type 2 diabetes.

\section{ABBREVIATIONS}

CKD, chronic kidney disease; SCI, silent cerebral infarction; MRI, magnetic resonance imaging; ACR, albumin-to-creatinine ratio; ADMA, asymmetric dimethylarginine; OHA, oral hypoglycemic agent; RAS, renin-angiotensin system.

\section{CONFLICT OF INTEREST}

The authors declare no conflict of interest.

1 National Kidney Foundation. K/DOQI clinical practice guidelines for chronic kidney disease: evaluation classification stratification. Part 4. Definition and classification of stages of chronic kidney disease. Am J Kidney Dis 2002; 39(Suppl 1): S46-S75.

2 Go AS, Chertow GM, Fan D, McCulloch CE, Hsu CY. Chronic kidney disease and the risks of death, cardiovascular events, and hospitalization. N Eng J Med 2004; 351: 1296-1305.

3 So WY, Kong AP, Ma RC, Ko GT, Kong AP, Zhao H, Luk AO, Lam CW, Ho CS, Tong PC, Chan JC. Glomerular filtration rate, cardiorenal end points, and all-cause-mortality in type 2 diabetic patients. Diabetes Care 2006; 29: 2046-2052.

4 Arnlöv J, Evans JC, Meigs JB, Wang TJ, Fox CS, Levy D, Benjamin EJ, D'Agostino RB, Vasan RS. Low-grade albuminuria and incidence of cardiovascular disease events in nonhypertensive and nondiabetic individuals: the Framingham Heart Study. Circulation 2005; 16: 969-975. 
5 Babazono T, Nyumura I, Toya K, Hayashi T, Ohta M, Suzuki K, Kiuchi Y, Iwamoto Y. Higher levels of urinary albumin excretion within the normal range predict faster decline in glomerular filtration rate in diabetic patients. Diabetes Care 2009; 32: 1518-1520.

6 Caramori ML, Fioretto P, Mauer M. The need for early predictors of diabetic nephropathy risk: is albumin excretion rate sufficient? Diabetes 2000; 49: 1399-1408.

7 American Diabetes Association. Nephropathy in diabetes. Diabetes Care 2004; 27 (Suppl 1): S79-S83.

8 Vermeer SE, Koudstaal PJ, Oudkerk M, Hofman A, Breteler MM. Prevalence and risk factor of silent brain infarcts in the population-based Rotterdam Scan Study. Stroke 2002; 33: 21-25.

9 Lee SC, Park SJ, Ki HK, Gwon HC, Chung CS, Byun HS, Shin KJ, Shin MH, Lee WR. Prevalence and risk factors of silent cerebral infarction in apparently normal adults. Hypertension 2000; 36: 73-77.

10 Fisher CM. Lacnar strokes and infarcts: a review. Neurology 1982; 32: 871-876.

11 Bruno A, Rosenberg GA. The spectrum of lacnar infarction in the elderly. Clin Geriatr Med 1991; 7: 443-453.

12 Kobayashi S, Okada K, Koide H, Bokura H, Yamaguchi S. Subcortical silent brain infarction as a risk factor for clinical stroke. Stroke 1997; 28: 1932-1939.

13 Naganuma T, Uchida J, Tsuchida K, Takemoto Y, Tatsumi S, Sugimura K, Nakatani T. Silent cerebral infarction predicts vascular events in hemodialysis patients. Kidney Int 2005; 67: 2434-2439.

14 Vermeer SE, Prins ND, den Heijer T, Hofman A, Koudstaal PJ, Breteler MM. Silent MRI infarcts and the risk of dementia and cognitive decline. N Engl J Med 2003; 348: 1215-1222.

15 Wada M, Nagasawa H, Kurita K, Koyama S, Arawaka S, Kawanami T, Tajima K, Daimon $\mathrm{M}$, Kato T. Microalbuminuria is a risk factor for cerebral small vessel disease in community-based elderly subjects. J Neurol Sci 2007; 15: 27-34.

16 Nakamura T, Kawagoe Y, Matsuda T, Ueda Y, Ebihara I, Koide H. Silent cerebral infarction in patients with type 2 diabetic nephropathy. Effects of antiplatelet drug dilazep dihydrochloride. Diabetes Metab Res Rev 2005; 21: 39-43.

17 Bouchi R, Babazono T, Nyumura I, Toya K, Hayashi T, Ohta M, Hanai K, Kiuchi Y, Suzuki K, Iwamoto $\mathrm{Y}$. Is reduced estimated glomerular filtration rate a risk factor for stroke in patients with type 2 diabetes? Hypertens Res 2009; 32: 381-386.

18 Uzu T, Kida Y, Shirahashi N, Harada T, Yamauchi A, Nomura M, Isshiki K, Araki SI, Sugimoto T, Koya D, Haneda M, Kashiwagi A, Kikkara R. Cerebral microvascular disease predicts renal failure in type 2 diabetes. J Am Soc Nephrol 2010; 21: 520-526.

19 Alberti KGMM, Zimmet P, for the WHO consultation. Definition, diagnosis, and classification of diabetes mellitus and its complications, part 2: diagnosis and classification of diabetes mellitus provisional report of a WHO consultation. Diabet Med 1998; 15: 539-553.
20 Hirsch AT, Haskal ZJ, Hertzer NR, Bakal CW, Creager MA, Halperin JL, Hiratzka LF, Murphy WR, Olin JW, Puschett JB, Rosenfield KA, Sacks D, Stanley JC, Taylor Jr LM, White CJ, White J, White RA, Antman EM, Smith Jr SC, Adams CD, Anderson JL, Faxon DP, Fuster V, Gibbons RJ, Hunt SA, Jacobs AK, Nishimura R, Ornato JP, Page RL, Riegel B. ACC/AHA 2005 Practice Guidelines for the Management of Patients With Peripheral Arterial Disease (Lower Extremity, Renal, Mesenteric, and Abdominal Aortic). Circulation 2006; 113: e436-e465.

21 Sasaki M, Hirai T, Taoka T, Higano S, Wakabayashi C, Matsusue E, Ida M. Discriminating between silent cerebral infarction and deep white matter hyperintensity using combinations of three types of magnetic resonance images: a multicenter observer performance study. Neuroradiology 2008; 50: 753-758.

22 Matsuo S, Imai E, Horio M, Yasuda Y, Tomita K, Nitta K, Yamagata K, Tomino Y, Yokoyama $\mathrm{H}$, Hishida A, Collaborators developing the Japanese equation for estimated GFR. Revised equations for estimated GFR from serum creatinine in Japan. Am J Kidney Dis 2009; 53: 982-992.

23 Friedewald WT, Levy RI, Fredrickson DS. Estimation of the concentration of low-density lipoprotein cholesterol in plasma, without use of the preparative ultracentrifuge. Clin Chem 1972; 18: 499-502.

24 Retnakaran R, Cull CA, Thorne KI, Adler AI, Holman RR; UKPDS Study Group. Risk factors for renal dysfunction in type 2 diabetes: U.K. Prospective Diabetes Study 74. Diabetes 2006; 55: 1832-1839.

25 O'Rourke MF, Safar ME. Relationship between aortic stiffening and microvascular disease in brain and kidney: cause and logic of therapy. Hypertension 2005; 46 : 200-204.

26 Ito S, Nagasawa T, Abe M, Mori T. Strain vessel hypothesis: a viewpoint for linkage of albuminuria and cerebro-cardiovascular risk. Hypertens Res 2009; 32: 115-121.

27 Pikula A, Böger RH, Beiser AS, Maas R, DeCarli C, Schwedhelm E, Himali JJ, Schulze F, Au R, Kelly-Hayes M, Kase CS, Vasan RS, Wolf PA, Seshadri S. Association of plasma ADMA levels with MRI markers of vascular brain injury: Framingham offspring study. Stroke 2009; 40: 2959-2964.

28 Hanai K, Babazono T, Nyumura I, Toya K, Tanaka N, Tanaka M, Ishii A, Iwamoto Y. Asymmetric dimethylarginine is closely associated with the development and progression of nephropathy in patients with type 2 diabetes. Nephrol Dial Transplant 2009; 24: $1884-1888$

29 Hashimoto J, Aikawa T, Imai Y. Large artery stiffening as a link between cerebral lacunar infarction and renal albuminuria. Am J Hypertens 2008; 21: 1304-1309.

30 Yokoyama H, Kawai K, Kobayashi M, Japan Diabetes Clinical Data Management Study Group. Microalbuminuria is common in Japanese type 2 diabetic patients: a nationwide survey from the Japan Diabetes Clinical Data Management Study Group (JDDM 10). Diabetes Care 2007; 30: 989-992. 